\title{
Chronic myelocytic leukemic fundus lesion: A case report
}

\author{
LE WEN XU, YINGHUA ZHANG，YAN LIU and YAN DING \\ Department of Ophthalmology, Rizhao People's Hospital, Rizhao, Shandong 276800, P.R. China
}

Received June 9, 2015; Accepted June 6, 2016

DOI: 10.3892/etm.2016.3577

\begin{abstract}
The current study reports a case of a patient with a chronic myelocytic leukemic fundus lesion, initially diagnosed in the Department of Ophthalmology, Rizhao People's Hospital (Rizhao, China). A male, 23-years-of-age, presented with a dark shadow in the front of the right eye, accompanied with blurred vision for 3 days (visual acuity of right eye, 0.4 ; visual acuity of left eye, 0.6 ). In addition, the patient had experienced gingival bleeding for 2 years, and recurrent upper respiratory infections for 1 year. A fundus examination revealed mild binocular papillary edema, dilated and tortuous veins, and the retina exhibited large quantities of scattered and dark red bleeding spots. The bleeding spots had white spots in the center, which exhibited typical Roth spots, whilst routine blood examination, abdominal ultrasound, marrow biopsy and other laboratory tests confirmed the diagnosis of chronic myelogenous leukemia. Patients with leukemia typically present with initial symptoms that include fever, fatigue, anemia and hepatosplenomegaly, and a diagnosis as a result of eye-related symptoms is rare, rendering the present case unique.
\end{abstract}

\section{Introduction}

Chronic myelocytic leukemia (CML), which is also known as chronic granulocytic leukemia, is a relatively rare malignant tumor that affects blood and bone marrow. CML is most common in older adults and among men. Based on the cases reported between 2009 and 2013, the number of new cases of CML was 1.8/100,000 individuals per year, whereas the number of deaths was $0.3 / 100,000$ individuals per year (1). These rates are age-adjusted, and the mortality rates from CML are highest among individuals aged 75-84 (1). Adequate supportive care and the initiation of imatinib mesylate have been essential in securing the recent favorable prognosis of patients with CML (2). CML is a clonal myeloproliferative

Correspondence to: $\mathrm{Mr}$. Le Wen $\mathrm{Xu}$, Department of Ophthalmology, Rizhao People's Hospital, 126 Taian Road, Rizhao, Shangdong 276800, P.R. China

E-mail: lwyhcn@163.com

Key words: chronic myelocytic leukemia, fundus lesion, young male disorder of hematopoietic stem cells characterized by the reciprocal translocation $\mathrm{t}(9 ; 22)$ ( $\mathrm{q} 34 ; \mathrm{q} 11)$, termed the Philadelphia chromosome $(\mathrm{Ph})(3)$. The resulting breakpoint cluster region-abelson 1 (BCR-ABL1) oncogene has deregulated tyrosine kinase activity (3), which produces a constitutive proliferative signal that is responsible for the transformed phenotype of CML cells. CML is estimated to account for $15-20 \%$ of adult leukemias (1). The age-adjusted annual incidence rate is 1.6 cases per 100,000 individuals, and the median age at diagnosis is 65 years, with a slight male predominance (1). CML is characterized by the generation of a large number of immature white blood cells that accumulate inside the bone marrow, and suppress the normal hematogenesis of bone marrow. In addition, the aforementioned cells are able to diffuse into the entire body through the blood circulation, leading to anemia, easy bleeding, infection and organ infiltration; thus, patients frequently present with such systemic manifestations, such as fever, fatigue, anemia and hepatosplenomegaly, as the initial symptoms. Consequently, the current case involving a patient with CML initially treated for visual impairment in the Department of Ophthalmology at the Rizhao People's Hospital (Rizhao, China), required the ophthalmologists to be familiar with the ocular manifestations of such diseases as CML, particularly the appearance of fundus lesions. Thus, ophthalmologists should be able to identify such diseases in order to reduce the rate of misdiagnosis and missed diagnosis of diseases in clinical work. The current authors treated a patient with CML with fundus lesion on December 4th 2011, and the diagnosis and treatment are reported herein.

\section{Case report}

The current study was conducted in accordance with the declaration of Helsinki, and was conducted with approval from the Ethics Committee of Rizhao People's Hospital. Written informed consent was obtained from the patient. A male patient, 23 years-of-age, visited the Department of Ophthalmology at the Rizhao People's Hospital as a result of a dark shadow in the front of the right eye, accompanied with blurred vision for 3 days, on December 4th, 2011. The patient had a history of occasional gingival bleeding for the previous 2 years, and a recurrent upper respiratory tract infection for 1 year; the latter of which was present 1 week prior to the patient's hospital visit. The patient's symptoms were improved following the oral administration of Bufferin caplets $(0.5 \mathrm{mg}$; Bristol-Myers Squibb Co., New York City, NY, USA); however, the patient 
still felt malaise. Systemic examination revealed mild anemia, and the skin and mucous membranes of the entire body indicated no jaundice or bleeding spots; however, several enlarged lymph nodes were palpable in the bilateral neck, and the liver could not be felt under the ribs. In addition, the spleen displayed swelling that reached four fingers under the rib. The remaining examinations indicated no concerning results. Eye examination provided the following results: Visual acuity of the right eye, 0.4, J3; visual acuity of left eye, $0.6, \mathrm{~J} 2$. The results of the intraocular pressure (IOP) assessment were as follows: Right eye, $19.6 \mathrm{mmHg}(1 \mathrm{mmHg}=0.133 \mathrm{kPa})$; left eye, $18.5 \mathrm{mmHg}$. The extraocular condition of both eyes was normal. Fundus examination revealed the following: Mild binocular papillary edema; the optic papilla boundary was less clear; the cup/disc ratio was 0.4 ; the retinal arteries exhibited no abnormalites; the veins were dilated tortuously; artery $:$ vein $=1: 2$; the central fovea of macula exhibited yellow patches and bleeding spots; the light reflex of the central fovea disappeared; and the entire retina exhibited a large quantity of scattered dark red bleeding spots, which were uneven in size, the smallest of which was equal to the vessel diameter, while the largest one was $\sim 1 / 4$ of the optic nerve's diameter. White spots were present in the center of bleeding spots, and exhibited the typical Roth spots (Figs. 1 and 2). Fundus fluorescein angiography identified the following: Numerous scattered bleeding spots on the binocular retina that covered the fluorescence; the center of the bleeding spots exhibited highly-fluorescent pale white spots (Figs. 3 and 4); the binocular midperiphery and the surrounding area had scattered capillary hemangioma-like high fluorescent spots (Figs. 5 and 6); the retinal veins were tortuous and dilated; and the macula lutea exhibited leakage of fluorescein. The results of laboratory assessments were as follows: White blood cell count, $420 \times 10^{9} / 1$; rod neutrophils, 13\%; segmented neutrophils, $49 \%$; promyelocytes, $2 \%$; myelocytes, 30\%; metagranulocytes, 2\%; hemoglobin, $84 \mathrm{~g} / 1$; platelet count, $544 \times 10^{9} / 1$, hematocrit, 0.242 ; sedimentation rate of erythrocyte, $18 \mathrm{~mm} / \mathrm{h}$; and lactate dehydrogenase, $860 \mathrm{IU} / \mathrm{l}$.

Thus, following the confirmation of a leukemia-caused fundus lesion, the patient was transferred to the Department of Hematology at the same institution for subsequent treatment. Abdominal ultrasound examination indicated that the left lobe of the liver was $8.5 \mathrm{~cm}$, the right lobe of the liver was $13.5 \mathrm{~cm}$, the echo was uniform and the vascular textures were clear; the spleen was obviously enlarged, with a spleen thickness of $7.0 \mathrm{~cm}$, and a length of $4.2 \mathrm{~cm}$ under the ribs. Bone marrow biopsy revealed that the proliferation of bone marrow cells was hyperactive, and the ratio of myeloid and erythroid cells was 37.8:1. The total number of myeloblasts was $92.5 \%$, among which the original myeloblasts accounted for $2 \%$, the promyelocytes accounted for $8 \%$, eosinophils accounted for $8 \%$, basophils accounted for $0.5 \%$ and myelocytes accounted for $22.5 \%$. Other cell types were inhibited; megakaryocytes demonstrated hyperactive proliferation, with $5 \%$ being mature cells, and platelet aggregation was also observed.

The patient was diagnosed with CML, and therefore, was admitted into the Department of Hematology for treatment with interferon $\left(3 \times 10^{6} \mathrm{IU} / \mathrm{m}^{2} / \mathrm{d}\right.$; Beijing Kawin Technology Co., Ltd., Beijing, China) via intramuscular injection thrice a week, oral hydroxyurea (30 mg/kg; Qilu Pharmaceutical, Co., Ltd., Shandong, China) twice a week, and imatinib mesylate

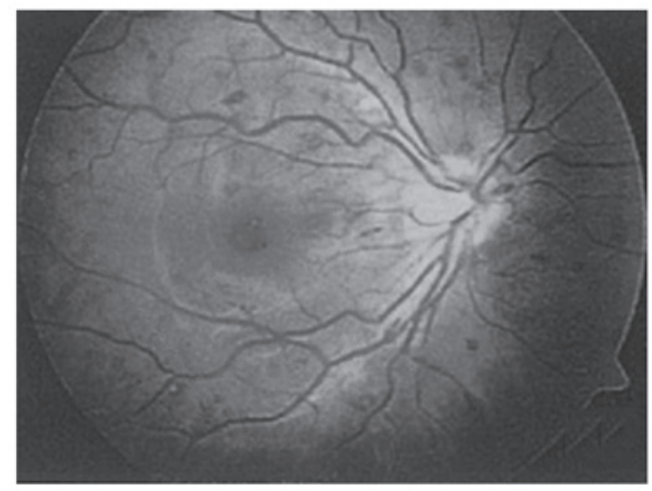

Figure 1. Right fundus image of the patient with a leukemia-induced fundus lesion. The discus opticus exhibited mild edema, the borders were undefined, the retina had several bleeding spots, with white spots in the center and bleeding in the macula lutea.

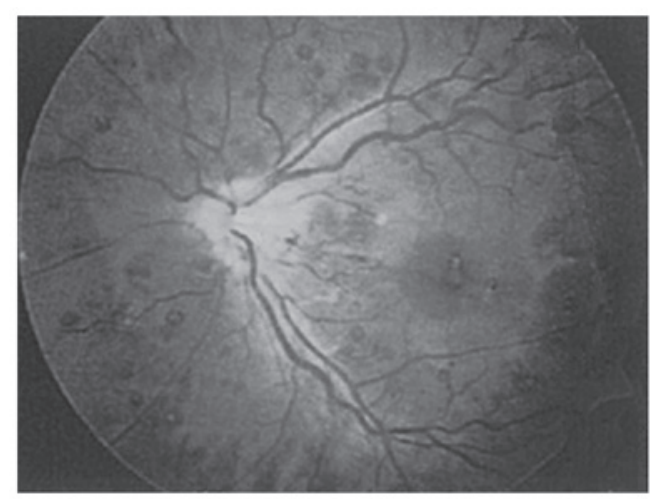

Figure 2. Left fundus image of the patient with a leukemia-induced fundus lesion, similar to that of the right fundus. The amount of bleeding exceeded that of the right eye.

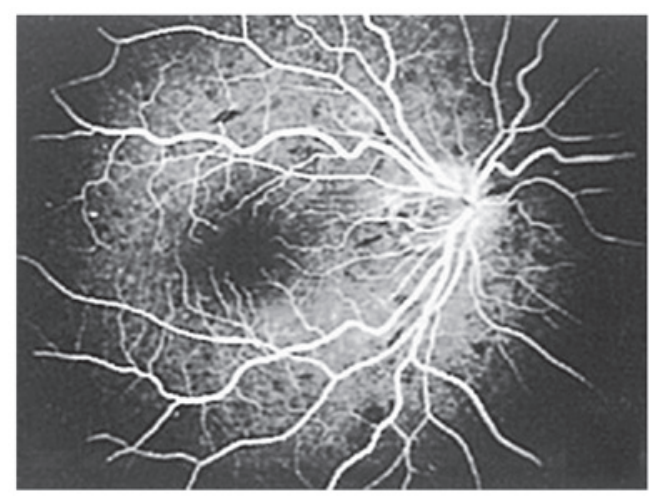

Figure 3. Right fundus fluorescein angiography of the patient with a leukemia-induced fundus lesion. The retina exhibited numerous scattered spots that covered the fluorescence. The center of the spots exhibited high fluorescence.

(400 mg; Chia Tai Tianqing Pharmaceutical Group Co., Ltd., Hong Kong, China) once daily. After 1 week the patient's condition was markedly improved, and a blood re-examination revealed the following: White blood cell count, 10x10\%/1; rod neutrophils, $12 \%$; segmented neutrophils, 53\%; myelocytes, $2 \%$; metagranulocytes, $2 \%$; hemoglobin, $84 \mathrm{~g} / 1$; and platelet count, $198 \times 10^{9} / 1$. Fundus examination revealed that the 


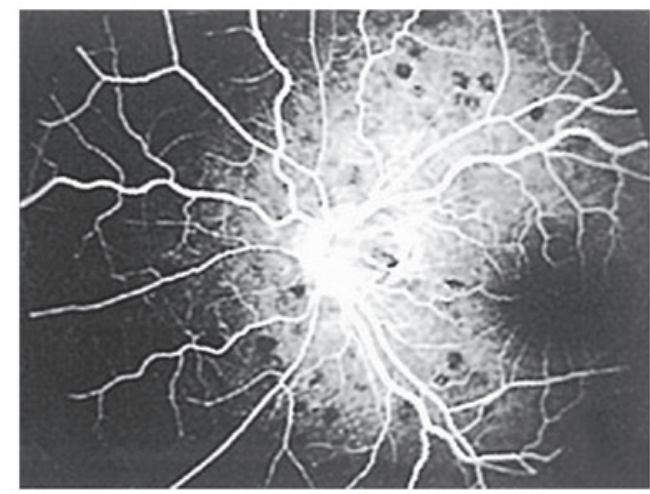

Figure 4. Left fundus fluorescein angiography of the patient with a leukemia-induced fundus lesion. The retina exhibited numerous scattered spots that covered the fluorescence. The center of the spots exhibited high fluorescence.

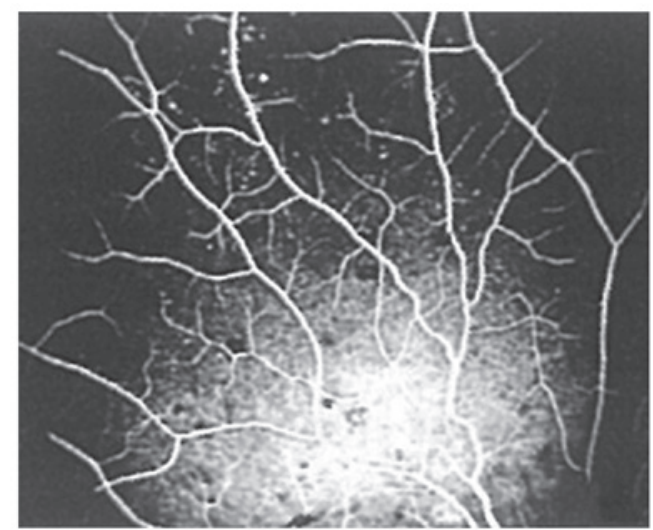

Figure 5. Right fundus fluorescein angiography of the patient with a leukemia-induced fundus lesion. The superior temple retina exhibited sporadic high fluorescence, suggesting the formation of capillary hemangioma.

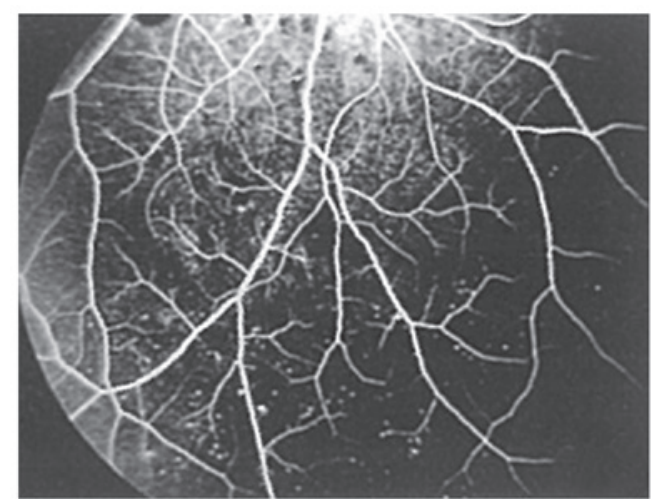

Figure 6. Left fundus fluorescein angiography of the patient with a leukemia-induced fundus lesion. The inferior retina exhibited a significant amount of dotted high fluorescence, suggesting the formation of capillary hemangioma.

small, sporadic and patch-like bleeding on the binocular eyeground had been removed, while a number of larger Roth spots remained. The patient remains in a stable condition under treatment with imatinib mesylate, although his dosage has decreased to $300 \mathrm{mg}$ once daily. Blood examinations, including routine blood analysis and hepatic and renal function are performed once a month. Bone marrow biopsy for bone marrow smear, and tests for the mutation in BCR-ABL kinase domain and chromosome are performed every 3-4 months. To date, the findings of these tests have demonstrated that the patient is in a stable condition.

\section{Discussion}

The natural history of CML in the absence of treatment is characterized by a triphasic course comprising of a chronic phase (CP)-CML, followed by an accelerated phase and invariably, progression to a final fatal blastic phase of an undefined duration of time (4). The prompt recognition of the patient's ocular fundus symptoms and early referral to oncologists for adequate management may have been a primary factor in determining the long-term outcome, as patients with $\mathrm{CP}-\mathrm{CML}$ at the time of diagnosis have the best long-term prognosis with treatment (4).

Previously, CML was incurable with conventional chemotherapy. However, the treatment strategies for CML have undergone revolutionary developments in the previous decade, predominantly due to an increased understanding of its molecular pathogenesis. The BCR-ABL1 fusion protein has been the target for drug design and therapies for CML, which have enabled the development of effective tyrosine kinase inhibitors (TKIs) that have the potential to eradicate the $\mathrm{Ph}$ chromosome-positive clone (2). Imatinib mesylate is a first generation TKI approved by the United States Food and Drug Administration in 2001, and has become the initial treatment of choice for the majority of patients with CML (2), as it results in a marked increase in the long-term survival and preservation of an acceptable quality of life (5).

Patients with leukemia typically present with fever, fatigue, anemia and hepatosplenomegaly as the initial symptoms, and eye-related symptoms are uncommon (6). Therefore, identification of leukemic patients is seldom a result of ocular inspections. The fundus of patients with leukemia may have lesions, which are generally more common in acute myelocytic leukemia (7). Abu el-Asrar et al (8) reported 74 cases of acute leukemia, which included 32 patients with retinopathy, accounting for $43 \%$ (8). A further study reported the prevalence as $35 \%$ (9). Thus, patients with the binocular tortuous and dilated retinal veins, and those with numerous Roth plaques, should be assessed for the possibility of leukemia. In the present study, the white spots in the patient's bleeding center were small, whereas had a greater quantity of blood been present, the patient may have been misdiagnosed with non-ischemic retinal vein occlusion. A case of leukemia-induced retinal vein occlusion has also been reported (10). Compared with the aforementioned case (leukemia-induced retinal vein occlusion), and the symptoms of the disease remitted subsequent to treatment, preventing the occurrence of retinal vein occlusion, the patient in the present study was identified to have CML at an earlier stage. It has been reported that the occurrence of central retinal vein thrombosis in patients with CML is not associated with an increase of blood viscosity, but is associated with the increase of anti-cardiolipin antibodies (10). This is notable as, although central retinal vein thrombosis is not a common symptom in CML, the fundal appearance of CML in the present case was similar to the appearance of central 
retinal vein thrombosis; therefore, comparable cases may be misdiagnosed as non-schemic retinal vein occlusion.

Roth spots are considered to be the characteristic pathological change of subacute bacterial endocarditis. Bleeding spots with a white center are a non-specific symptom caused by the rupture of retinal capillaries and the aggregation of fibrins and platelets. Clinically, numerous diseases are associated with the presence of Roth spots, which are predominantly detected in patients with subacute bacterial endocarditis, septicemia, toxoplasmosis, AIDS, leukemia, diabetes, hypertension, vasculitis and traumatic brain injuries in infants. Therefore, upon the detection of Roth spots, clinicians must perform further assessments to identify the relevant disease (11-14).

The occurrence of retinal hemorrhage in patients with leukemia is associated with a significant reduction in hemoglobin and hematocrit, while an increase in the number of leukocytes is significantly associated with the appearance of Roth spots (8). The patient in the present case exhibited hemoglobin levels of $84 \mathrm{~g} / 1$, whilst the hematocrit decreased to 0.242 , and the white blood cell count increased to $420 \times 10^{9} / 1$. In addition, the binocular retina had a large number of bleeding spots, and all bleeding spots had a white center. Specchia et al (15) reported that patients with leukemia who had retinopathy exhibited an increased level of blood lactate dehydrogenase, with an average of 812 IU/1, whilst patients who did not have retinopathy displayed a mean blood lactate dehydrogenase level of 607 IU/1. The blood lactate dehydrogenase level of the current patient was increased to $860 \mathrm{IU} / 1$ (normal range, 100-300 IU/1), which was consistent with the literature (15). With regards to the prognosis of CML, certain studies have reported that acute leukemic patients over 40 years-of-age with cotton wool spots in the fundus had a worse prognosis, and the impact of cotton wool spots was greater than that of age in terms of prognosis (9). Retinopathy is an important clinical manifestation that determines poor prognosis in patients in the remission period. The complete remission rate and survival rate of young patients who do not have retinopathy are significantly higher than those of older patients with retinopathy (15). CML in the patient in the current study was detected at an early stage, and no cotton wool spots were observed in the fundus. Subsequent to treatment, the retinal hemorrhage was reduced, the white blood cell count was significantly decreased and the systematic symptoms were significantly improved. This indicates a good prognosis.

In summary, ophthalmologists should consider a thorough systemic evaluation of patients with binocular tortuous and dilated retinal veins, in addition to those with several Roth plaques, as this may be an initial indicator of CML. As a corollary, the authors suggest to clinicians that all patients with newly diagnosed leukemia of any type be referred for ocular examination, regardless of whether they are symptomatic. A timely diagnosis, adequate supportive care and the initiation of imatinib mesylate have been essential for securing a favorable prognosis in the current patient.

\section{References}

1. Howlader N, Noone AM, Krapcho M, Neyman N, Aminou R, Waldron W, Altekruse SF, Kosary CL, Ruhl J, Tatalovich Z, et al (eds). SEER Cancer Statistics Review, 1975-2009 (Vintage 2009 Populations), National Cancer Institute Bethesda, MD (seer.cancer.gov/csr/1975_2009_pops09/). Accessed September 2013.

2. Baccarani M,Cortes J,Pane F,NiederwieserD, Saglio G, Apperley J, Cervantes F, Deininger M, Gratwohl A, Guilhot F, et al: Chronic myeloid leukemia: An update of concepts and management recommendations of European Leukemia Net. J Clin Oncol 27: 6041-6051, 2009

3. Quintás-Cardama A and Cortes J: Molecular biology of bcr-abl1-positive chronic myeloid leukemia. Blood 113: 1619-1630, 2009.

4. Cortes J: Natural history and staging of chronic myelogenous leukemia. Hematol Oncol Clin North Am 18: 569-584, 2004.

5. Gambacorti-Passerini C, Antolini L, Mahon FX, Guilhot F, Deininger M, Fava C, Nagler A, Della Casa CM, Morra E, Abruzzese E, et al: Multicenter independent assessment of outcomes in chronic myeloid leukemia patients treated with imatinib. J Natl Cancer Inst 103: 553-561, 2011.

6. Huang PK and Sanjay S: Visual disturbance as the first symptom of chronic myeloid leukemia. Middle East Afr J Ophthalmol 18: 336-338, 2011.

7. Vrcek I, Finnerty K, Ford P, Hogan RN and Mancini R: Relapsing acute lymphoblastic leukemia presenting with a rapidly enlarging and vision-threatening orbital mass. Int Ophthalmol 35: 257-260, 2015.

8. Abu el-Asrar AM, al-Momen AK, Kangave D, Harakati MS and Ajarim DS: Correlation of fundus lesions and hematologic findings in leukemic retinopathy. Eur J Ophthalmol 6: 167-172, 1996.

9. Abu el-Asrar AM, al-Momen AK, Kangave D and Harakati MS: Prognostic importance of retinopathy in acute leukemia. Doc Ophthalmol 91: 273-281, 1995-1996.

10. Al-Abdulla NA, Thompson JT and La Borwit SE: Simultaneous bilateral central retinal vein occlusion associated with anticardiolipin antibodies in leukemia. Am J Ophthalmol 132: 266-268, 2001.

11. Fred HL: Little black bags, ophthalmoscopy and the Roth spot. Tex Heart Inst J 40: 115-116, 2013.

12. Ling R and James B: White centered retinal haemorrhages (Roth spots). Postgrad Med J 74: 581-582, 1998.

13. Falcone PM and Larrison WI: Roth spots seen on ophthalmoscopy: Diseases with which they may be associated. Conn Med 59: 271-273, 1995.

14. Reddy SC and Jackson N: Retinopathy in acute leukaemia at initial diagnosis: Correlation of fundus lesions and haematological parameters. Acta Ophthalmol Scand 82: 81-85, 2004.

15. Specchia G, Albano F, Guerriero S, Buquicchio C, Pomes L, Pastore D, Carluccio P, Delle Noci N and Liso V: Retinal abnormalities in newly diagnosed adult acute myeloid leukemia. Acta Haematol 105: 197-203, 2001. 